\title{
PECULIARITIES OF THE LINK MOTION.
}

By A. Dunbar, Second Assistant Fngineer U. S. N.

THe link motion, as applied to a numerous class of engines for screw vessels in the Navy, is the type upon which the following deductions are based:

To render them more simple, they will be divided into four parts, viz.:-First, Radius of Link. Second, Variation of Lead. Third, Point of Suspension. Fourth, Effect of the point of attachment of the excentric rods to the link upon the point of cutting off.

First. The radius of the link is correct, when the lead increases or decreases equally upon both ends of the stroke throughout the movement of the link. With the dimensions commonly used, it is the distance from the centre of the link-block to the centre of the excentric, less three quarters of the lap and lead of the valve. Reference to this will be made in the second part of this paper, in order to dispense with a diagram to illustrate this point.

Second. The lead increases, when the link is moved from full gear into mid gear, if the excentric rods are crossed; when the crank is on the centre next to the cylinder, and decreases, if they are not crossed under the same circumstances.

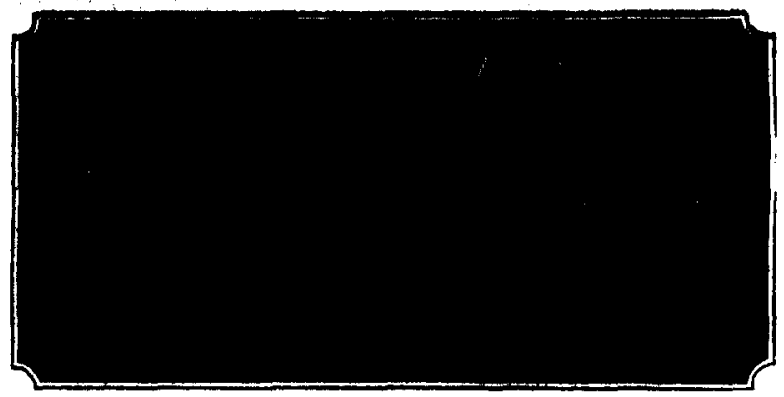

In Fig. 2, $A$ and $B$, are the positions of the centres of the for. ward and back motion excentrics, when the crank is on the centre, c, next to the cylinder. The direction in which the crank moves forward, is indicated by the arrows.

The full lines represent the position of the excentric rods and link, in full gear forward motion, crank on centre, c, and the broken lines, the position of the same in mid gear, crank on centre, c. By inspection, it will be seen, that when the link is drawn up into mid gear, the valve has opened the outboard port, and increased 
the lead equal to the distance, $x$. In these diagrams, the valve is supposed to be without lead, when the link is in full gear. The uneven broken lines represent the position of the excentric rods and link in full gear, forward motion, crank on centre, $\mathrm{c}^{\prime}$, and the even broken lines, the position of the same in mid gear, crank on centre, $\mathrm{c}^{\prime}$. By inspection, it will be seen, that when the link is drawn up into mid gear, the valve has opened the inboard port, and increased the lead equal to the distance, $\mathbf{x}^{\prime}$; as $\mathrm{X}$ and $\mathbf{X}^{\prime}$, are equal, the radius of the link, is correct. If they were not equal, it would be necessary to divide the difference, and through that point and the extremities of the link, draw a curve, the radius of which will be the true radius for the link.

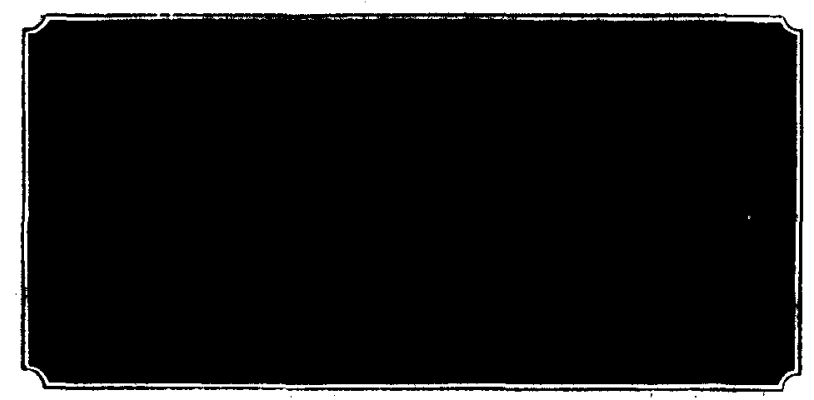

In Fig. 3, $A$ and $B$, are the positions of the centres of the forward and back motion excentrics, when the crank is on the centre, c. The full lines represent the position of the excentric rods and link in full gear, forward motion, crank on centre, $\mathrm{c}$, and the broken lines, the pasition of the same in mid gear, crank on centre, $c$. When the link is lowered into mid gear, it will be seen, that the valve has moved over tho outboard port, and the lead is a negative quantity equal to the distance, $\mathbf{x}$. The uneven broken lines represent the position of the link and excentric rods in full gear, forward motion, crank on centre, $\mathrm{c}^{\prime}$, and the even broken lines, the position of the same in mid gear, crank on centre, $\mathrm{c}^{\prime}$. As on the other centre, when the link is lowered into mid gear, the valve has moved over the inboard port, and the lead is a negative quantity equal to the distance, $\mathbf{x}^{\prime}$.

In Fig. 2, the excentric rods are crossed at $o$, when the crank is on the centre next to the cylinder, and in Fig. 3 , they are not crossed.

Vox. LV.—THIRd SRRIER.-No. 6.-JUNR, 1868. 
Third. The paint of suspension should be so arranged, that the slip upon the link-block should be the least obtainable in practice, that the wear upon these parts may be as slight as possible, for they are generally so constructed, that the lost motion in these parts can only be taken up by substituting a new block.

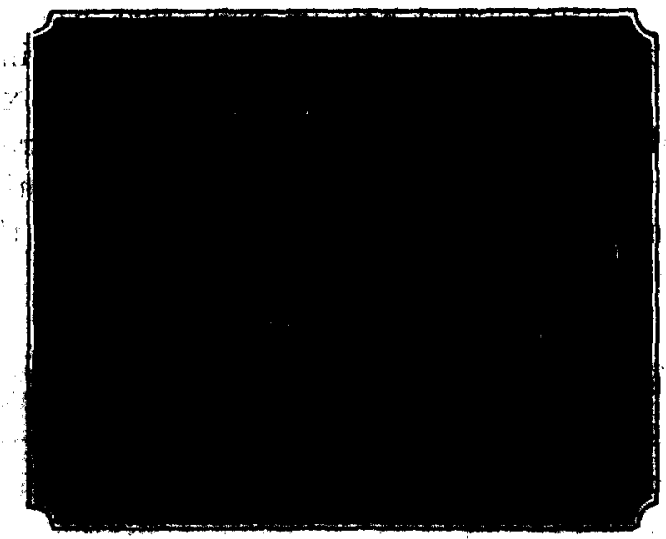

If the point, $D$, (Fig. 4,) moves in an arc whose concave side is up, as is ordinarily the case, and as there represented, the point, $\mathrm{t}$, will, if the drawing be enlarged eight times, develop a figure as shown at Fig. 5. If the point, $\mathrm{E}$, moves in a straight line without slip upon the block throughout a revolution of the crank, a figure will be developed by the point, $D$, as shown at Fig. 6 (enlarged as before). If the point, D, Fig. 7, be moved outboard upon the linksaddle, about three-quarters of the lap and lead of the valve, it

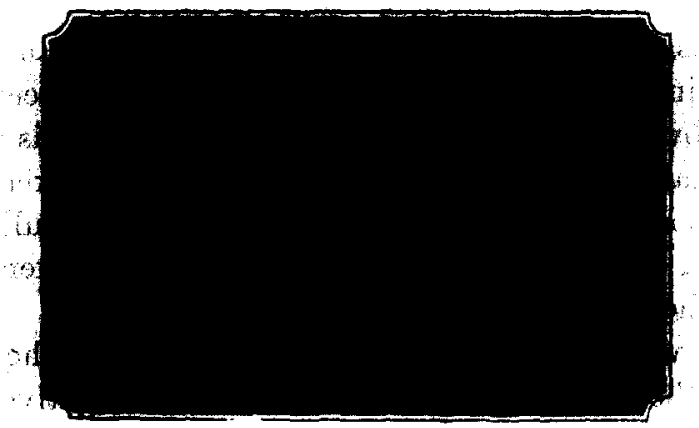

will then develop, if the point, $E$, moves in a straight line, and the drawing be enlarged as before, a figure as shown at Fig. 9. This figure is very nearly the arc of a circle. 
Find the centre of the are that nearest approximates this figure, and we have the position of the extremity of the reversing arm, and the length of the suspension rod.

If the point, $D$, moves in that approximate arc, the point, $E$, will develop a figure as shown at Fig. 8. As the convex side of Fig. 9 is up, the extremity of the reversing arm must be below the link, and almost under the point, $D$, vertically.

With these points in this position; the slip of the link upon the block, will not exceed one-fortieth the travel of the valve. The distance the point, $\mathbf{D}$, has to be moved outboatd upon the link. saddle varies with every variation of the length of the link com. pared to the travel, and lap and lead of the valve. The author is unable to give any rule for flnding this point that he can rely on in all cases. To explain the process for laying down the figure shown at Fig. 6 , would extend this paper beyond its intended limit. The distance is determined from the shape of this figure, and this varies with every change in the dimensions. Those given here are for the general proportion of the "Juniata" class of engines. The slip increases as the link is moved either way from the given position, and these points should be located for the position in which the link is mostly used. The extremity of the reversing arm must be in the centre of the approximate arc, for if this point does not coincide with that centre, the slip is materially increased.

Fourth. In engines using a slide-valve with lap and lead, the point of cutting off is generally unequal if the lead is equal, owing to the angularity of the connecting rod. If the excentric rods are attached to the link at a point inboard from the centre of the

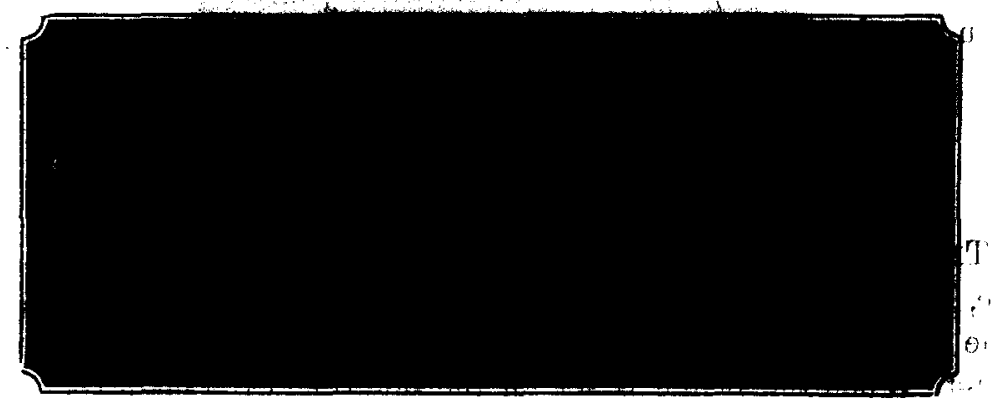

curvature of the link, as at $\rightarrow$, Fig. 4 , and the engine be back-acting, this arrangement will equalize the point of cutting off, provided the point, $\mathrm{a}$, be the proper distance from the centre of the link- 
block. To illustrate this, reference will be made to Fig. 10. Suppose the point of cutting off, to be at six-tenths the stroke, and the length of the connecting rod to be two and one-half times the stroke of the piston, then the crank will stand at the point, $\mathbf{B}$, when cutting off on the outboard end, and at $\mathrm{H}^{\prime}$, when cutting off on the inboard end of the cylinder.

The crank has moved through more than six-tenths of a semirevolution, when it arrives at $H$, and as the valve moves with the crank, the valve would be more than closed, were it not for the angle, E, G, A, formed by the link and excentric rods at this time, which angle shortens the distance from $\mathbf{E}$ to $\mathbf{A}$, and prevents the valve from elosing until the crank arrives at $\mathrm{H}$.

Again, when the crank arrives at $\mathbf{H}^{\prime}$, it has not completed sixtenths of a semi-revolution, and the valve would not have closed, were it not for the angle, $\mathrm{E}^{\prime}, \mathrm{G}^{\prime}, \mathrm{A}^{\prime}$, formed by the link and excentric rods at this time, which angle shortens the distance from $\mathbf{E}^{\prime}$ to $\mathbf{A}^{\prime}$, and closes the valve when the crank arrives at $\mathbf{H}^{\prime}$. (The point of cutting off, on the "Juniata's" engines, is within an eighth of an inch of being equal.) With the valve attached to the link, and moving coincident with it, if the engine was direet-acting, the difference in the point of cutting off' would be nearly doubled; but if a rocker-shaft be interposed between the link and valve, the difference is equalized.

In the preceding parts of this paper, this peculiarity of the link motion was not taken into consideration, because the back motion, excentric rod, and end of the link makes nearly the same angle as the forward motion, at the same time, and the result is to draw the link bodily inboard, and does not materially affect the deductions then stated.

\section{A. F. BALLAS' PATENT R. R. SWITCH.}

THe many accidents which occur through the switches now in use, leave no doubt that they are highly defective and dangerous. The principal objections against them are-

1st. When the switch is set for the side track, the main track is interrupted. Thus, if a switch has been left standing for the side track by the switch-tender, trains which follow the main track will be thrown from the rails. 